\title{
Rhamnolipids from Pseudomonas aeruginosa Rn19a Modifies the Biofilm Formation over a Borosilicate Surface by Clinical Isolates
}

\author{
Jair Carrazco-Palafox ${ }^{1,2}$ (), Blanca Estela Rivera-Chavira ${ }^{1}$, Jaime Raúl Adame-Gallegos ${ }^{1}(\mathbb{D}$, \\ Luz María Rodríguez-Valdez ${ }^{1} \mathbb{D}$, Erasmo Orrantia-Borunda ${ }^{3}$ and Guadalupe Virginia Nevárez-Moorillón ${ }^{1, * \mathbb{D}}$ \\ 1 Facultad de Ciencias Químicas, Universidad Autónoma de Chihuahua, Circuito Universitario s/n Campus \\ Universitario II, 31125 Chihuahua, Mexico; jcarrazco@uach.mx (J.C.-P.); bchavira@uach.mx (B.E.R.-C.); \\ jadame@uach.mx (J.R.A.-G.); lmrodrig@uach.mx (L.M.R.-V.) \\ 2 Facultad de Medicina y Ciencias Biomédicas, Universidad Autónoma de Chihuahua, Circuito No. 1, \\ Campus Universitario II, 31125 Chihuahua, Mexico \\ 3 Centro de Investigación en Materiales Avanzados Av. Miguel de Cervantes Saavedra 120, \\ Complejo Industrial Chihuahua, 31136 Chihuahua, Mexico; erasmo.orrantia@cimav.edu.mx \\ * Correspondence: vnevare@uach.mx; Tel.: +52-614-236-6000
}

Citation: Carrazco-Palafox, J.; Rivera-Chavira, B.E.; AdameGallegos, J.R.; Rodríguez-Valdez, L.M.; Orrantia-Borunda, E.; NevárezMoorillón, G.V. Rhamnolipids from Pseudomonas aeruginosa Rn19a Modifies the Biofilm Formation over a Borosilicate Surface by Clinical Isolates. Coatings 2021, 11, 136. https://doi.org/10.3390/ coatings11020136

Academic Editor: Mariana Carmen Chifiriuc

Received: 28 December 2020

Accepted: 22 January 2021

Published: 27 January 2021

Publisher's Note: MDPI stays neutral with regard to jurisdictional claims in published maps and institutional affiliations.

Copyright: (c) 2021 by the authors. Licensee MDPI, Basel, Switzerland. This article is an open access article distributed under the terms and conditions of the Creative Commons Attribution (CC BY) license (https:// creativecommons.org/licenses/by/ $4.0 /)$.
Abstract: Microbial cells are reversibly associated with surfaces in the form of biofilms. Adhesion is the mechanism used by the microorganisms to bind to a surface initially; no biofilm is formed without the initial adhesion. The aim of this work was to evaluate the efficacy of the rhamnolipids of Pseudomonas aeruginosa Rn19a in inhibiting the biofilms formed by the clinical isolates Escherichia coli I5, Pseudomonas aeruginosa E26, Enterococcus faecalis I27 on borosilicate coupons inside a Center for Disease Control and Prevention (CDC) reactor. The isolate E26 (P. aeruginosa) did not show an adverse effect on biofilm formation by the rhamnolipid presence and showed normal growth in all the conditions tested (dynamic and static growth). The Enterococcus faecalis I27 isolate decreased its biofilm formation ability in $2.2 \log \mathrm{CFU} / \mathrm{cm}^{2}$ in static conditions by the addition of rhamnolipids and $3.0 \log$ units in dynamic conditions. Finally, the E. coli I5 isolate was more susceptible to the influence of the borosilicate coupon covered with rhamnolipids. E5 reduced its biofilm formation capacity by $3.0 \log \mathrm{CFU} / \mathrm{cm}^{2}$ units at static conditions by the rhamnolipid addition and $6.0 \mathrm{log}$ units at dynamic conditions. Biofilm formation was also observed by Confocal Laser Scanning Microscopy. In summary, the application of rhamnolipids may be useful to prevent the initial adhesion of bacteria to borosilicate surfaces. At a minimum, rhamnolipids effectively inhibit or diminish adhesion to surfaces by biofilm-forming isolates that do not belong to the genus Pseudomonas.

Keywords: Pseudomonas aeruginosa; rhamnolipids; biofilms; microbial adhesion; bioreactor; borosilicate surface

\section{Introduction}

In aquatic environments, usually bacteria are growing as biofilms. Biofilm can be defined as a sessile community of microorganisms irreversibly linked to a substrate or interface or with each other; the microbial cells are integrated into an array of extracellular polymeric substances (EPS) produced by the community an altered phenotype in growth rate and genotype [1,2].

The life cycle of biofilms includes the adhesion of individual cells to a surface, followed by the production of EPS that covers the cells and allows the formation of colonies, that when fully formed, can lead to the release of few cells in planktonic phenotypes, that can then be dispersed in the surrounded area. Nutrients and oxygen have a gradient from the outside to the center of biofilms, and this condition modifies the metabolic activity and duplication time of biofilm-forming microbial cells. In addition, cells inside are more 
tolerant of antibiotics [2,3]. Biofilms are exceedingly difficult to remove and are, therefore, a cause of concern in medicine and industrial settings, among other environments [4].

On the other hand, if it is considered that without the initial step in biofilm formation, these microbial communities cannot be formed [5], inhibition of microbial adhesion is the most logical option to prevent its presence $[4,6]$. Surface treatment or modification of surface properties using surfactants has been proposed to inhibit microbial adhesion [6-8].

Surfactants are amphiphilic compounds containing hydrophobic and hydrophilic moieties, which reduce surface and interfacial tension $[9,10]$. Biological surfactants are structurally heterogeneous molecules, and several microorganisms can produce them as part of their metabolism [11]. Microbial biosurfactants are classified by their chemical structure as well as by their origin and, based on their action, can be identified as biosurfactants or bioemulsifiers [12].

Biosurfactants produced by bacteria are the most studied surfactants. Although there are different biosurfactants produced by bacteria, rhamnolipids are among the most extensively reported compounds. Extracellular rhamnolipids are mainly produced by Pseudomonas spp. [13-15], and although there are different reports of rhamnolipids, all of them possess a similar chemical structure [16]. Rhamnolipids contain one or two units of L-rhamnose, linked to one or two units of $\beta$-hydroxy alkanoic acid tails of varying lengths $[10,11]$. While additional rhamnose confers more hydrophilicity to the rhamnolipids, the additional carbons in the acid chains can increase their hydrophobicity [17].

Most bacteria release biosurfactants to dissolve hydrophobic substrates and facilitate the absorption and assimilation of hydrocarbons, but also, it has been demonstrated their antiadhesive properties $[6,18]$. Biosurfactants have the potential to modify the properties of surfaces and interfere with the initial adhesion of microorganisms, and, therefore, prevent the formation of biofilms. The introduction of new imaging modalities has helped in the conceptual understanding of biofilms. The most significant advance in our understanding of biofilms came after the invention of confocal laser scanning microscopy (CLSM) [19,20].

Rhamnolipids produced by P. aeruginosa have potential as antiadhesive and dispersant agents that could represent a promising approach to prevent the initial adhesion of bacteria and fungi to surfaces while forming biofilms $[7,21]$. The influence of rhamnolipids on biofilm formation is of importance at early stages but also on the conservation or dispersal of mature biofilm structure [20]. The effect of rhamnolipids in biofilm formation can be observed in biofilms formed by non-Pseudomonas bacterial strains as well [19]. Albeit its potential, there are not enough studies on the anti-adherence properties of rhamnolipids [20]. The use of a CDC Bioreactor can provide similar conditions to test static and dynamic conditions.

High concentrations of rhamnolipids could have an antiadhesive effect against all reported microorganisms, although the specific antiadhesive effect depends on the microorganism analyzed. Therefore, this work was aimed to evaluate the effectiveness of Pseudomonas aeruginosa Rn19a rhamnolipids on the inhibition of biofilm formation on borosilicate coupons, using a CDC bioreactor. The effect of the rhamnolipid was tested against clinical isolates with multiple antibiotic resistance, with differential capacity in biofilm formation.

\section{Materials and Methods}

\subsection{Microbial Strains and Culture Conditions}

Pseudomonas aeruginosa Rn19a was isolated from soil samples in northern Mexico that had been contaminated with used mobile oil. The microorganism was previously identified as a producer of lipases and biosurfactants [22,23]. Bacterial clinical isolates Escherichia coli I5, Pseudomonas aeruginosa E26, and Enterococcus faecalis I27 were also used. The three clinical isolates were obtained from patients with urinary tract infections and have been characterized as multidrug-resistant and strongly adherent to glass and polystyrene surfaces [24]. 
Bacterial strains were maintained in Nutrient Agar (BIOXON, Mexico City, Mexico) tube slants at $4{ }^{\circ} \mathrm{C}$. For experiments, microbial growth was obtained in Tryptic Soy Broth (TSB) (BIOXON, Mexico City, Mexico) incubated at $30^{\circ} \mathrm{C}$ to obtain a bacterial suspension equal to $10^{8}$ cells $/ \mathrm{mL}$, adjusted by optical density at $595 \mathrm{~nm}$ [25].

$\mathrm{Rn} 19 \mathrm{a}$ isolate was analyzed by the $16 \mathrm{~S}$ rRNA gene sequence. The DNA extraction was done from $2 \mathrm{~mL}$ bacterial culture via the phenol-chloroform extraction protocol. Total DNA was quantified and stored at $-20^{\circ} \mathrm{C}$. DNA samples were amplificated by PCR using 16SF AGAGTTTGATCCTGGCTCAG and 16SR ACGGCTACCTTGTTACGACTT primers. Each reaction was done in a final volume of $50 \mu \mathrm{L}$ containing $29 \mu \mathrm{L}$ of sterile Milli Q water, $7 \mu \mathrm{L}$ of $10 \times$ Taq buffer, $4 \mu \mathrm{L}$ of each primer, $1 \mu \mathrm{L}$ of dNTP Mix, $1 \mu \mathrm{L}$ of Taq Polymerase, and 4 $\mu \mathrm{L}$ of extracted genomic DNA $(100 \mathrm{ng} / \mu \mathrm{L})$. The conditions for PCR amplification included an initial denaturation step $\left(95^{\circ} \mathrm{C}, 10 \mathrm{~min}\right), 25$ amplification cycles of denaturation $\left(93^{\circ} \mathrm{C}\right.$, $1 \mathrm{~min}$ each), annealing $\left(50^{\circ} \mathrm{C}, 1 \mathrm{~min}\right.$ each), elongation $\left(72{ }^{\circ} \mathrm{C}, 1: 30 \mathrm{~min}\right.$ each), and final extension step $\left(72{ }^{\circ} \mathrm{C}, 10 \mathrm{~min}\right)$. PCR products were analyzed and purified in agarose gel electrophoresis $(1.5 \%)$. The PCR product was sequenced by Psomagen (Rockville, MD, USA) by next-generation sequencing methodology. The sequence was compared with $16 \mathrm{~S}$ rDNA gene sequences selected from GenBank, using BLAST by multiple alignments using CLUSTALX2 v2.1. The software used for the phylogenetic analysis were Mega-X v10.0.5, and Unipro UGENE v33, using 1000 repetitions in the bootstrap analysis [24].

\subsection{Biosurfactant Production and Characterization}

P. aeruginosa Rn19a was grown with agitation $(175 \mathrm{rpm})$ at $37^{\circ} \mathrm{C}$ for 9 days (Innova 4300, New Brunswick Shaker Incubator, Edison, NJ, USA) in an Erlenmeyer flask (500 mL) containing $400 \mathrm{~mL}$ M9 mineral medium (initial $\mathrm{A}_{600 \mathrm{~nm}}$ of the culture was between 0.020 and 0.025 , using sterile medium as the reference) added with $0.5 \%$ dextrose [22]. After incubation, culture medium was centrifuged $\left(3200 \times g, 24^{\circ} \mathrm{C}, 20 \mathrm{~min}\right.$ ) (Eppendorf Centrifuge 570R, Hamburg, Germany) to obtain cell-free supernatant (CFS) and the cell pellet [26-28]. Rhamnolipid was recovered by solvent-extraction from CFS. CFS was deproteinized by heating at $110{ }^{\circ} \mathrm{C}$ for $10 \mathrm{~min}$. After cooling, it was acidified to $\mathrm{pH} 3.0$ with $2 \mathrm{~N} \mathrm{HCl}(v / v)$; the acidified supernatant was maintained overnight at $4{ }^{\circ} \mathrm{C}$ for the complete precipitation of biosurfactant. Rhamnolipids were separated by continuous extraction with equal amount of ethyl acetate in a separating funnel at room temperature. The ethyl acetate was evaporated from the sample using a rotary evaporator [26,29]. Additionally, the rhamnolipid was resuspended with chloroform-ethanol-water, 2:1:1 mixture (Chloroform: J.T. Baker SOLUSORB; Ethanol: Faga Lab, Mocorito, Sin. Mexico), vigorously vortexed, and centrifuged $(10,000 \times g, 10 \mathrm{~min})$ to accelerate the phase separation. The mixture formed three phases. The upper phase contained mainly water and waste from the culture broth. The middle phase, which is the thinnest, was a layer colored with pigments. Both layers were removed separately and transferred to a clean bottle. Further these fractions were extracted three times with chloroform-ethanol (2:1) to complete recovery of biosurfactant. The lower phase containing the biosurfactant was collected in a clean container. For this fraction, the solvent was removed using a rotary evaporator. Additionally, the dried product was washed three times with absolute ethanol to complete the removal of the residual pigments [30]. A viscous honey-colored rhamnolipid product was collected after solvent evaporation. Samples were lyophilized for further analysis. Biosurfactant was analyzed by thin-layer chromatography (TLC) [31], and the biosurfactant activity of the cell-free supernatant was verified by surface tension by the capillary method $[32,33]$.

\subsection{Biofilm Formation Using the CDC Bioreactor}

A CDC bioreactor (BioSurface Technologies Corp., Bozeman, MT, USA) of $1 \mathrm{~L}$ capacity was used to assay the biofilm formation by the clinical isolates Escherichia coli I5, Pseudomonas aeruginosa E26, and Enterococcus faecalis I27. Borosilicate glass (BS) coupons (BioSurface Technologies Corp., Bozeman, MT, USA) for biofilm formation with $12.7 \mathrm{~mm}$ of diameter and thickness of $3.8 \mathrm{~mm}$ designed for CDC bioreactor were used. A set of 
sterile and clean coupons were immersed in $0.4 \%(w / v)$ biosurfactant solution. Then, the coupons were incubated for $24 \mathrm{~h}$ at $35^{\circ} \mathrm{C}$ and then washed three times with sterile water. Two series of coupons were prepared: the first treated with biosurfactants and the second untreated [6].

Two conditions were tested, a static incubation and a dynamic model simulated by mechanical agitation of the bioreactor. For the static model, the CDC bioreactor was filled with $300 \mathrm{~mL}$ of sterile TSB and a set of clean, sterile coupons. For each experiment, the reactor was inoculated with $100 \mu \mathrm{L}$ at $10^{8}$ cells $/ \mathrm{mL}$ of one of the clinical strains; the incubation temperature was $35^{\circ} \mathrm{C}$. At 12 -h intervals $(0,12,24,36,48,60$, and $72 \mathrm{~h})$, biofilmcoated coupons were extracted for analysis. In addition, biofilms were compared on coupons treated and nontreated with rhamnolipids (Figure 1)

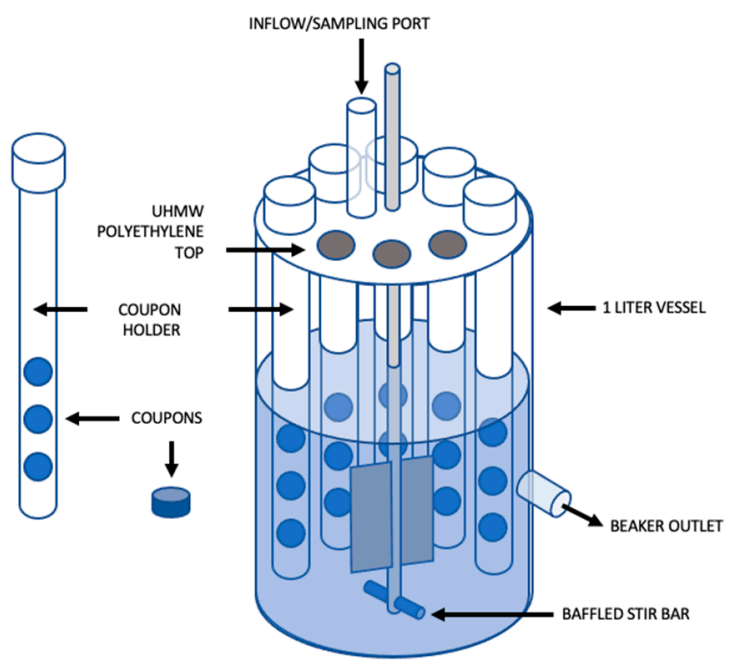

Figure 1. CDC Bioreactor for biofilm formation.

For biofilm formation on a dynamic model, the following conditions were incorporated: sterile TSB was continuously added to the bioreactor at a rate of $2.7 \mathrm{~mL} / \mathrm{min}$ using a peristaltic pump, and the bioreactor was incubated at $35{ }^{\circ} \mathrm{C}$ with mechanical agitation (stirring bar, in a magnetic plate) at $60 \mathrm{rpm}$. These conditions were based on the use of CDC Bioreactor for biofilm formation, according to the EPA Method MB-19-05 [34]. Three independent experiments were done for each condition.

For both static and dynamic conditions, coupons were aseptically removed at the specified times and were gently immersed in $10 \mathrm{~mL}$ of sterile peptone water to remove planktonic cells. Coupons were scraped with the edge of a sterile stainless-steel spatula; then, the spatula was rinsed in $9 \mathrm{~mL}$ of water with sterile peptone. After scraping, the coupons were rinsed with $1 \mathrm{~mL}$ of water with peptone to remove any remaining biofilm and complete a final volume of $10 \mathrm{~mL}$ in the dilution tube. The sample was stirred in vortex for $1 \mathrm{~min}$ to breakdown the biofilm groups. Decimal serial dilution of disintegrated biofilm sample was carried out, and dilutions were inoculated by pour plate technique into Standard Plate Count Agar (SPC) (BIOXON, Mexico City, Mexico). Plates were incubated at $35{ }^{\circ} \mathrm{C}$ for $18-24 \mathrm{~h}$, and the number of colony-forming units (CFU) was calculated for each coupon side.

\subsection{Biofilm Analysis by Confocal Laser Microscopy}

After biofilm formation, coupons were carefully removed. Coupons were rinsed and dyed with 100 L of BacLight $^{\mathrm{TM}}$ Live/Dead staining kit (Invitrogen, Carlsbad, CA, USA), following the manufacturer's instructions. Biofilms were rinsed and mounted into the confocal microscope. The stained cell aliquots were observed in an inverted CLSM (LSM 700 Zeiss, Oberkochen, Germany) with a Plan-Apochromat 20×/0.8 objective (Carl Zeiss AG, Oberkochen, Germany). Detection was done using excitation with two lasers at $488 \mathrm{~nm}$ 
and $561 \mathrm{~nm}$, and the fluorescence emission was collected from 500 to $550 \mathrm{~nm}$ and from 570 to $700 \mathrm{~nm}$. Images were scanned at $600 \mathrm{~Hz}$ and analyzed with the software of the equipment (ZEN imaging software, version 2011, black edition. Carl Zeiss, MicroImaging, Jena, Germany).

\section{Results}

\subsection{Molecular Identification of Selected Pseudomonas Strain}

$16 \mathrm{~S}$ rRNA gene sequence was used as a taxonomic analysis. The 16S rRNA gene sequence allows differentiation between organisms, including species and subspecies levels. The strain Rn19a was found to share $97.26 \%$ similarity with P. aeruginosa SK4 and other P. aeruginosa strains. Strain P. aeruginosa Rn19a has been assigned the GenBank access number MN176395 (Figure 2).

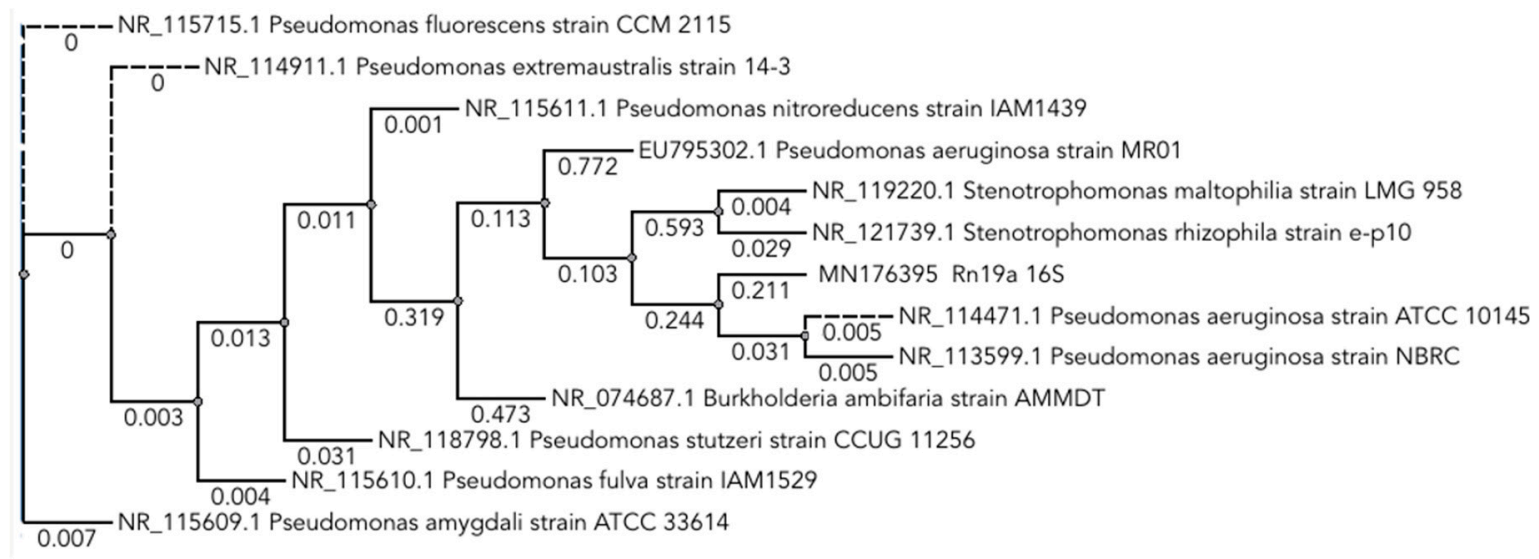

Figure 2. Phylogenetic tree based on the $16 \mathrm{~S}$ rRNA gene sequences of the strain Pseudomonas aeruginosa Rn19a. This analysis includes sequences of related genera from GenBank. Rn19a is included with the reference number provided by GenBank.

\subsection{Biosurfactant Purification and Functionality.}

Rhamnolipids from P. aeruginosa Rn19a were obtained from the cell-free culture medium, as described in Section 2.2. The semipurified rhamnolipid was a viscous honeycolored product, with a yield of $12 \mathrm{mg} / \mathrm{L}$. Results from the TLC proved that the rhamnolipid was a mixture of mono- and dirhamnolipids (data not shown) [17]. The lowest surface tension reached by the aqueous solution of rhamnolipids $(0.5 \% \mathrm{w} / \mathrm{v})$ was $16.9 \mathrm{mN} / \mathrm{m}$ against pure water $(72.1 \mathrm{mN} / \mathrm{m})$ at $20^{\circ} \mathrm{C}$.

\subsection{Biofilms Formation in Static and Dynamic Conditions in the CDC Bioreactor}

The attachment of the rhamnolipid to the borosilicate surface was initially tested in glass slides, with different concentrations of rhamnolipids under static conditions (data not shown). The surface conditioning and rhamnolipid concentration were selected based on reports where the lowest concentration demonstrates a reduction in adhesion $[6,35]$.

Microbial biofilm formation by the three clinical isolates in the borosilicate coupons (BS) in the static mode (S), without rhamnolipids (RHA), showed an average bacterial growth, as observed in Figure 3. Results demonstrated that there were no limitations in bacterial adhesion and biofilm formation in the $72 \mathrm{~h}$-incubation period.

Biofilm formation kinetics for Escherichia coli I5 is included in Figure 3. There are two trends observed; first, biofilm growth in the absence of rhamnolipids (I5-S-BS and I5-D-BS), i.e., E. coli $\mathrm{I} 5$ showed a lower growth curve associated with the removal of biomass under dynamic conditions (I5-D-BS) without it being a blunt difference. On the other hand, when the BS coupon was covered with rhamnolipids (I5-S-BS-RHA and I5-D-BS-RHA), microbial counts were smaller with up to three logarithmic units of difference. Even more, under 
dynamic conditions (I5-D-BS-RHA), cells attached to the BS coupons were in the range of 1-2 $\log \mathrm{CFU} / \mathrm{cm}^{2}$ after $72 \mathrm{~h}$ of incubation.

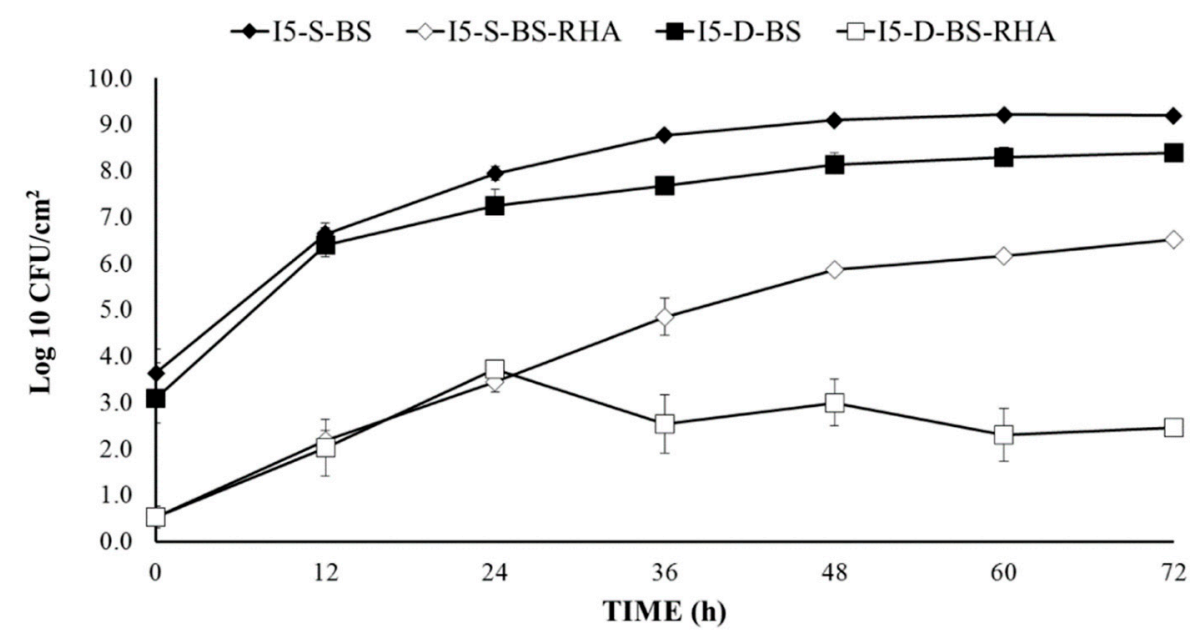

Figure 3. Biofilm formation kinetics of Escherichia coli I5 on borosilicate (BS). The microbial count is expressed in $\log _{10} \mathrm{CFU} / \mathrm{cm}^{2}$. The parameters observed were static (S) or dynamic (D) and borosilicate (BS) model. Coupons were treated with rhamnolipids (RHA) or without them (without notation).

Figure 4 shows the imagen obtained by CLSM of the BS coupons with E. coli I5 growth after $72 \mathrm{~h}$ of incubation under the conditions described above. Figure 4A (I5-S-BS) shows the growth of E. coli 15 grouped into growing colonies (green) with a few dead spots (red). In Figure $4 \mathrm{~B}$, the size of the colonies is smaller and with a higher proportion of dead cells. In both cases, microbial adhesion to the BS surface was obtained in the absence of rhamnolipids. In contrast, the presence of rhamnolipids decreased the number of colonies on the coupon surface. The dead (red) colonies were observed in higher proportions (see Figure 4C), while in Figure 4D, a reduction on the number of colonies is observed.
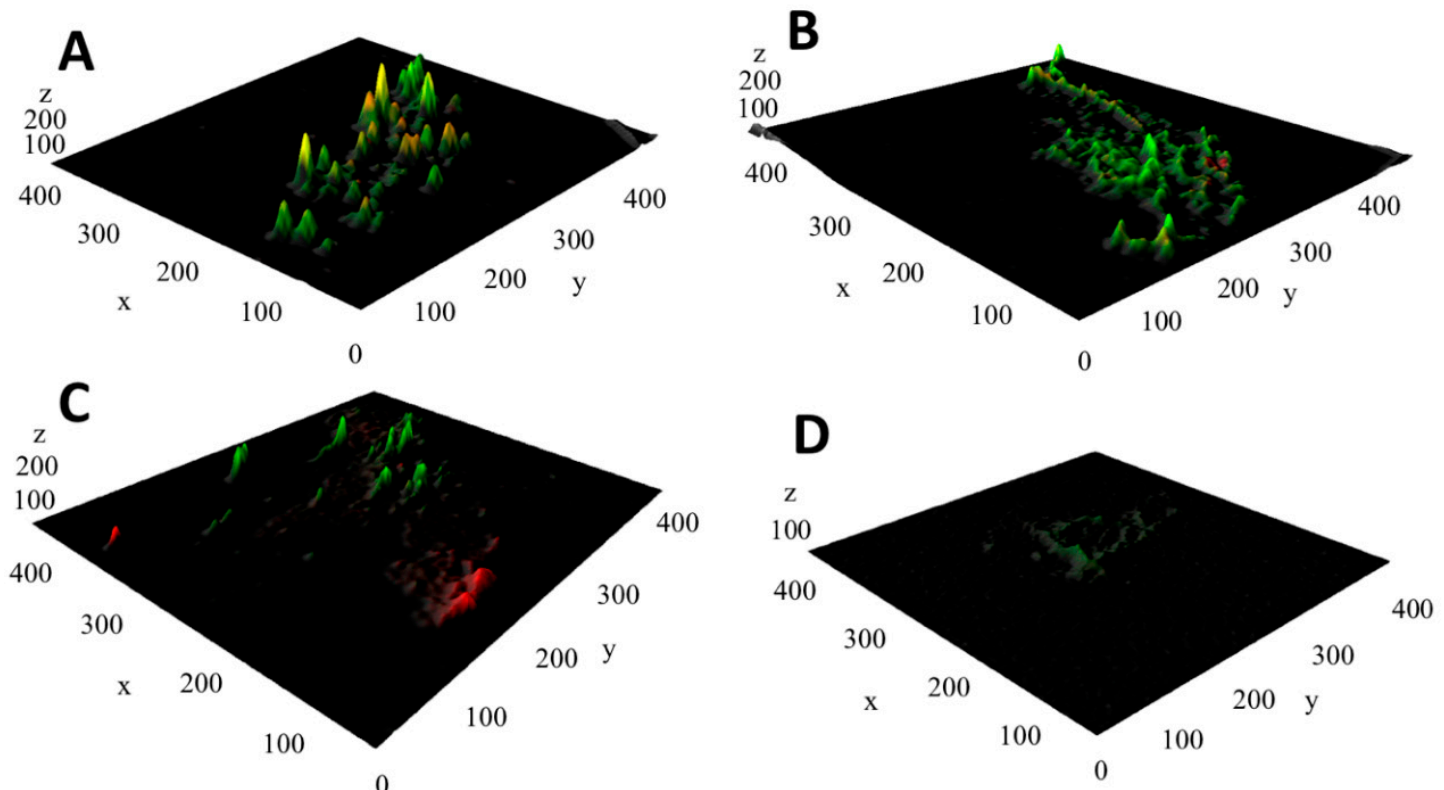

Figure 4. Analysis of Escherichia coli I5 biofilms by Confocal Laser Scanning Microscopy. (A) I5-S-BS. (B) I5-D-BS. (C) I5-S-BS-RHA. (D) I5-D-BS-RHA. The parameters observed were static (S) or dynamic (D) models, borosilicate surface (BS). Coupons were treated with rhamnolipids (RHA) or without them (without notation). The analysis shows the results after $72 \mathrm{~h}$ incubation. The scale on the graphs is microns. 
In contrast, growth for the Pseudomonas aeruginosa E26 isolate was not affected by the different conditions tested (Figure 5). Growth kinetics was similar when the coupons were treated with rhamnolipids or when the bioreactor was agitated. Therefore, rhamnolipids did not interfere with the adhesion of the microbial cells to BS but did not favor it either; shear stress did not affect the adherence of microbial cells or biofilm formation.

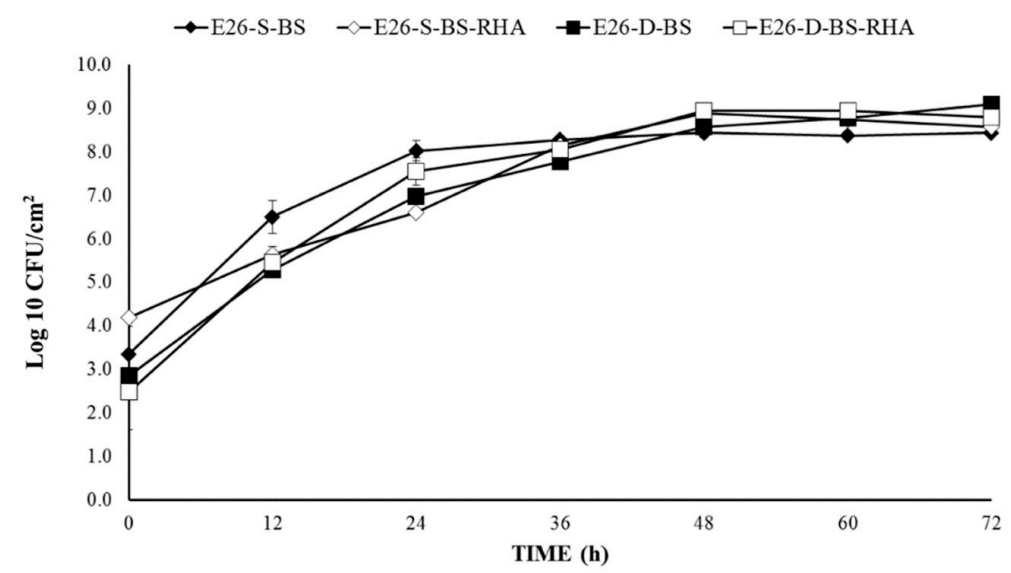

Figure 5. Biofilm formation kinetics of Pseudomonas aeruginosa E26 on borosilicate (BS). The microbial count is expressed in $\log 10 \mathrm{CFU} / \mathrm{cm}^{2}$. The parameters observed were static (S) or dynamic (D), borosilicate (BS) model. Coupons were treated with rhamnolipids (RHA) or without them (without notation).

When observed under the microscope (CLSM), Pseudomonas aeruginosa E26 showed only metabolically active zones, with no dead cells or colonies identified by the green only areas in Figure 6. In Figure 6A, no isolated colonies were distinguished, as presented by a mature biofilm. Shear stress in the dynamic model (Figure 6B) decreased the area with microbial growth. The colony shape was mostly elongated or laxer in the presence of rhamnolipids and static conditions (Figure 6C). Finally, in the BS coupon covered with rhamnolipids and under dynamic conditions, colonies were smaller (Figure 6D). It is important to mention that in $\mathrm{C}$ and $\mathrm{D}$, biofilm floccules were observed on the medium.
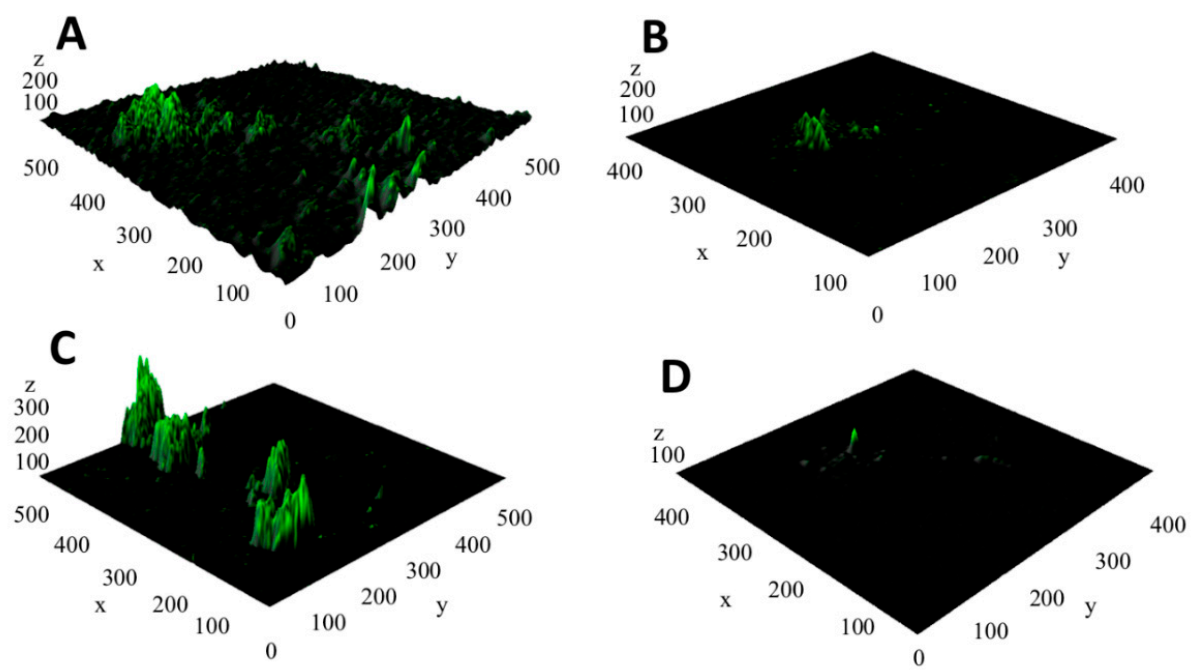

Figure 6. Analysis of Pseudomonas aeruginosa E26 biofilms by Confocal Laser Scanning Microscopy. (A) I5-S-BS. (B) I5-D-BS. (C) I5-S-BS-RHA. (D) I5-D-BS-RHA. The parameters observed were static (S) or dynamic (D) models, borosilicate surface (BS). Coupons were treated with rhamnolipids (RHA) or without them (without notation). The analysis shows the results after $72 \mathrm{~h}$ incubation. The scale on the graphs is microns. 
Regarding the clinical isolate Enterococcus faecalis I27, two tendencies were also observed, like the ones described for E. coli I5. The first includes kinetics in the absence of rhamnolipids (I27-S-BS and I27-D-BS). E. faecalis I27 showed a lower growth curve associated with the removal of biomass under dynamic conditions (I27-D-BS). When the BS coupons were covered with rhamnolipids (I27-S-BS-RHA and I27-D-BS-RHA), microbial growth was lower, and the maximum number of cells was lower in more than three logarithmic units after $72 \mathrm{~h}$ of incubation (Figure 7).

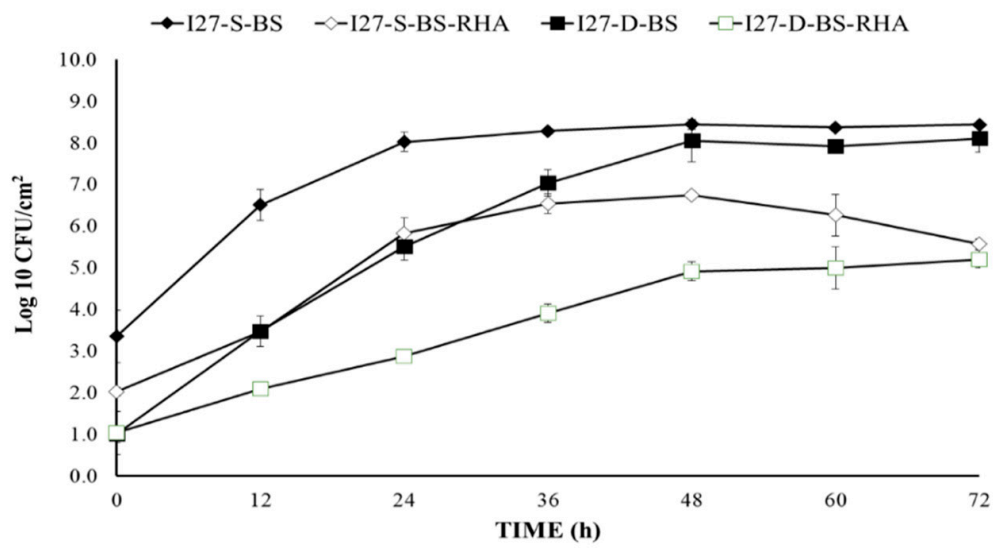

Figure 7. Biofilm formation kinetics of Enterococcus faecalis I27 on borosilicate (BS). The microbial count is expressed in $\log 10 \mathrm{CFU} / \mathrm{cm} 2$. The parameters observed were static (S) or dynamic (D), borosilicate (BS) model. Coupons were treated with rhamnolipids (RHA) or without them (without notation).

Figure 8 shows the microscopic analysis of Enterococcus faecalis I27 biofilm formed on the BS coupons after 72 of incubation. In Figure 8A, a large number of colonies are observed, but in some cases, there are zones of dead cells (red zones). The number of colonies diminishes in the dynamic model (Figure 8B). The addition of rhamnolipid on the surface of the coupons affected the maximum number of cells incorporated in biofilms; therefore, in Figure 8C, there are clear zones and areas with dead cells. Additionally, Figure $8 \mathrm{D}$ shows active colonies along with dead zones.
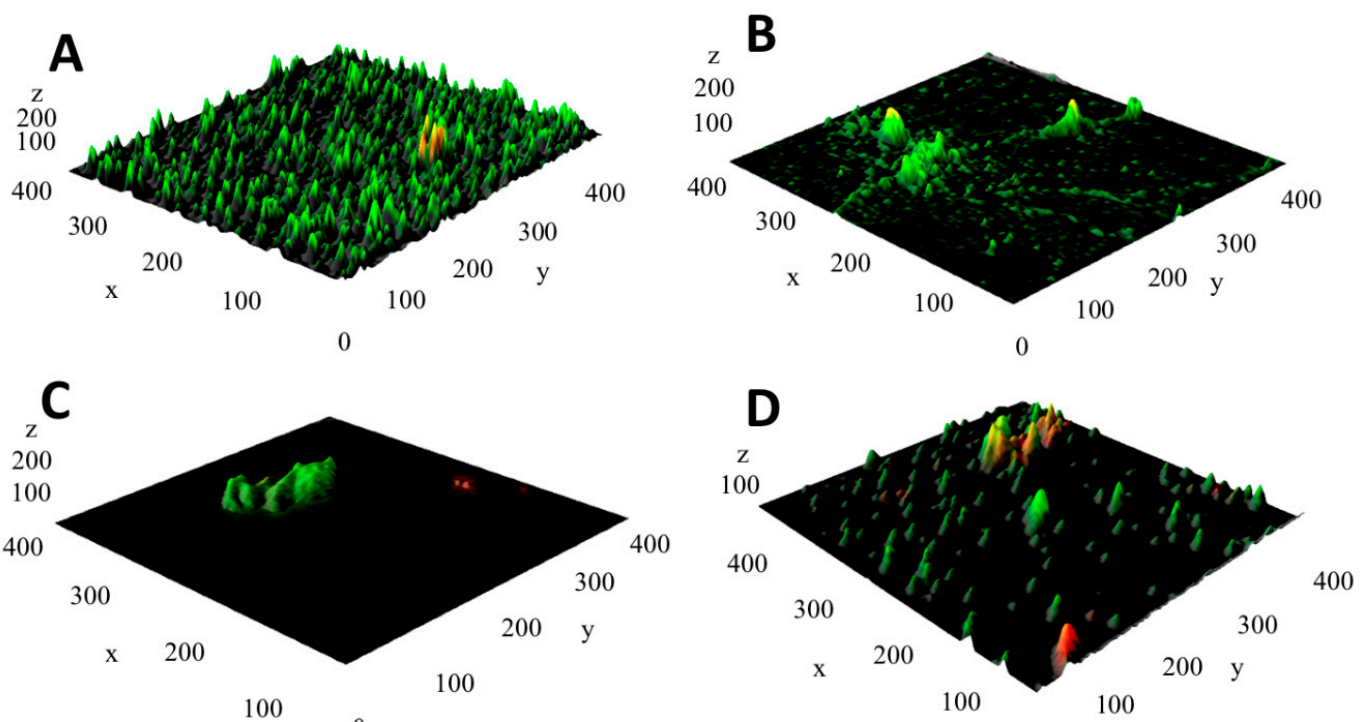

Figure 8. Analysis of Enterococcus faecalis I27 biofilms by Confocal Laser Scanning Microscopy. (A) I27-S-BS. (B) I27-D-BS. (C) I27-S-BS-RHA. (D) I27-D-BS-RHA. The parameters observed were static (S) or dynamic (D) models, borosilicate surface (BS). Coupons were treated with rhamnolipids (RHA) or without them (without notation). The analysis shows the results after $72 \mathrm{~h}$ incubation. The scale on the graphs is microns. 


\section{Discussion}

Most of our results show an inhibitory effect of rhamnolipids on biofilms, which is similar to other reports $[8,19,36]$. The treatment of coupons with rhamnolipids $(0.4 \%$ $w / v)$ showed some changes in biofilms formation. In the case of E. coli I5, BS coupons covered with rhamnolipids diminished the initial adhesion so that there was a decrease in more than 3 logarithmic units after $72 \mathrm{~h}$ of incubations, as compared with coupons without rhamnolipids. Previous reports suggest that rhamnolipids can promote swarming, mediate nutrient uptake, and disrupt biofilm architecture and cell surface polarity [37]. Rhamnolipids also have antimicrobial activity against other bacteria. In the E. coli 15 biofilm model, confocal microscopy revealed different dead zone profiles in the absence or presence of rhamnolipids; this suggests that $E$. coli $\mathrm{I} 5$ has difficulty growing into borosilicate coupons. Furthermore, the addition of a surfactant inhibited the development of the biofilm. Finally, the combination of rhamnolipids plus agitation (Figure 4D) achieved the highest reduction in microbial growth.

For the P. aeruginosa E26 strain, the addition of rhamnolipids to the BS surface did not present an adverse effect on biofilm formation. The strain maintained a typical growth with and without rhamnolipids; the same behavior has been reported before [38]. When P. aeruginosa E26 biofilm was observed with the confocal laser microscope, microbial growth was evident in the presence or absence of rhamnolipids. In dynamic conditions, despite the fact that the growth was similar in all cases, bacterial distribution on BS coupon was less dense. It has been reported that in dynamic conditions, biofilms with porous structures are presented, while in static conditions, Pseudomonas spp. exhibit comparatively uniform biofilms [39]. Since the addition of rhamnolipids (Figure 6C) favored the height of the biofilms formed, it is suggested that the surfactant contributes to a more relaxed consistency, detachment, and bacterial dispersion. Rhamnolipids may be involved in reducing extracellular polymeric substances (EPS) and the detachment of microcolonies [25].

Enterococcus faecalis I27 was also affected by the presence of rhamnolipids, where biofilm formation was reduced. The lag and exponential phases of the growth curve were lagged in the presence of the biosurfactant (Figure 7). Additionally, E. faecalis I27 presented a slight decrease in microbial count between 48 and $72 \mathrm{~h}$. In the specific case of this strain, confocal laser microscopy showed a further reduction in growth as well as areas of dead colonies in the presence of rhamnolipids. It is suggested that a higher concentration of biosurfactant can inhibit the formation of new biofilms or the release of preformed ones.

When dynamic conditions were added, but the coupons were not covered with rhamnolipids, the main modification to microbial growth was presented at early stages of the microbial growth curve. The stirring speed and the change of culture medium favored the decrease in initial adhesion but did not prevent biofilm formation. P. aeruginosa E26 strain had a slightly good exponential phase along with E. coli I5. Regarding P. aeruginosa E26, it could produce rhamnolipids in the initial phase, which might be associated with the formation of biofilms [38]. In addition, at the stationary phase, rhamnolipids along with shear rate can be responsible for biofilm biomass detachment [20]. As mentioned earlier, E. coli I5 showed P. aeruginosa E26-like growth in the dynamic model. However, E. faecalis I27 had the lowest growth curve of the three microorganisms studied; also, in static conditions, it had the lowest growth during the first $36 \mathrm{~h}$ of incubation.

It has been suggested that rhamnolipids have a fundamental role in biofilm formation in Pseudomonas spp. The biological roles assigned to rhamnolipids in biofilm-forming Pseudomonas strains include the formation of structures with pores and channels and dispersion from mature biofilms [39]. On the other hand, rhamnolipids also have antimicrobial action reported against other bacteria, such as Staphylococcus aureus [19] or sulfate-reducing bacteria [36]. The differences observed in the biofilm formation and inhibition in the three clinical isolates reported in this study also show a differential effect of the rhamnolipid produced by P. aeruginosa Rn19a on the P. aeruginosa strain and the E. coli and Enterococcus faecalis strains. 
To better understand the effect of the rhamnolipid coating on different surfaces, changes in the surface characteristic need to be better described. Differences in biofilm formation among the bacterial strains can be related to the physicochemical properties of the cell surface. It has been proposed that the cells' hydrophobicity is correlated to the initial adhesion to a particular surface, and growth conditions can modify the bacterial cell surface [40]. Therefore, modifications on the physicochemical properties of a surface will modify the capacity for an initial adhesion of microbial cells, as the initial step on biofilm formation.

The effect of rhamnolipids on polystyrene demonstrated an increase in hydrophobicity and Lewis acid-base properties as compared with the control; this effect was not observed in a stainless-steel surface [40]. In another report, however, the polystyrene surface coated with rhamnolipid was less hydrophobic [41]. The chemical structure and physicochemical properties of the rhamnolipid used can also influence the coating properties; as with many biologically derived molecules, the standardization of the compounds is necessary to assure the expected result.

The deleterious effects of bacterial and fungal biofilms are evident in the food industry, in environmental applications such as biofouling, and in medicine since persistent biofilms are responsible for many recurrent infections [2]. The use of coating materials to promote a biofilm-resistant surface is of particular importance in the material used for medical implants, including metal or plastic-based devices [8,42]. Biosurfactants (particularly, rhamnolipids) can be used as a coating material, since it reduces biofilm formation [37] and has also been shown to disrupt previously grown biofilms $[18,19,27]$. Applications that include a coating of medically related surfaces with rhamnolipids, including catheters and implants, require further information on the toxicity of the biosurfactants or the effect of these molecules on the immunological system. In particular, with rhamnolipids, the pathogenicity of the bacterial strain can be an obstacle for the use of their metabolites. Biosurfactant production and purification are also major challenges [10], so that synthetic or modified molecules have been proposed $[7,39]$ for control of biofilm formation.

Therefore, rhamnolipids produced by P. aeruginosa Rn19a can be proposed as a coating material for the control of biofilm formation by non-Pseudomonas bacterial strains. Rhamnolipids are more effective at inhibiting or decreasing adhesion to surfaces by strains that form biofilms that do not belong to the genus Pseudomonas. In contrast, Pseudomonas strains that produce rhamnolipids are less susceptible.

Author Contributions: Conceptualization, J.C.-P., J.R.A.-G. and G.V.N.-M.; data curation, J.C.-P., L.M.R.-V. and G.V.N.-M.; formal analysis, B.E.R.-C. and L.M.R.-V.; investigation, J.C.-P.; methodology, E.O.-B. and G.V.N.-M.; supervision, B.E.R.-C., J.R.A.-G. and E.O.-B.; writing-original draft, J.C.-P.; writing - review and editing, G.V.N.-M. All authors have read and agreed to the published version of the manuscript.

Funding: This work was partially supported by the School of Chemical Sciences, Universidad Autónoma de Chihuahua and CIMAV.

Institutional Review Board Statement: Not applicable.

Informed Consent Statement: Not applicable.

Data Availability Statement: No new data were created or analyzed in this study. Data sharing is not applicable to this article.

Acknowledgments: Jair Carrazco-Palafox acknowledges the School of Medicine and Biomedical Sciences, Universidad Autónoma de Chihuahua for supporting his doctoral studies.

Conflicts of Interest: The authors declare no conflict of interest.

\section{References}

1. Donlan, R.M. Biofilms: Microbial Life on Surfaces. Emerg. Infect. Dis. 2002, 8, 881-890. [CrossRef] [PubMed]

2. Satpathy, S.; Sen, S.K.; Pattanaik, S.; Raut, S. Review on bacterial biofilm: An universal cause of contamination. Biocatal. Agric. Biotechnol. 2016, 7, 56-66. [CrossRef] 
3. Høiby, N.; Ciofu, O.; Johansen, H.K.; Song, Z.Z.; Moser, C.; Jensen, P.Ø.; Molin, S.; Givskov, M.; Tolker-Nielsen, T.; Bjarnsholt, T. The clinical impact of bacterial biofilms. Int. J. Oral Sci. 2011, 3, 55-65. [CrossRef] [PubMed]

4. Verderosa, A.D.; Totsika, M.; Fairfull-Smith, K.E. Bacterial biofilm eradication agents: A current review. Front. Chem. 2019, 7, 824. [CrossRef] [PubMed]

5. Lebeaux, D.; Ghigo, J.-M.; Beloin, C. Biofilm-Related Infections: Bridging the Gap between Clinical Management and Fundamental Aspects of Recalcitrance toward Antibiotics. Microbiol. Mol. Biol. Rev. 2014, 78, 510-543. [CrossRef]

6. Zeraik, A.E.; Nitschke, M. Biosurfactants as Agents to Reduce Adhesion of Pathogenic Bacteria to Polystyrene Surfaces: Effect of Temperature and Hydrophobicity. Curr. Microbiol. 2010, 61, 554-559. [CrossRef]

7. Aleksic, I.; Petkovic, M.; Jovanovic, M.; Milivojevic, D.; Vasiljevic, B.; Nikodinovic-Runic, J.; Senerovic, L. Anti-biofilm properties of bacterial di-rhamnolipids and their semi-synthetic amide derivatives. Front. Microbiol. 2017, 8, 2454. [CrossRef]

8. Ceresa, C.; Tessarolo, F.; Maniglio, D.; Tambone, E.; Carmagnola, I.; Fedeli, E.; Caola, I.; Nollo, G.; Chiono, V.; Allegrone, G.; et al. Medical-grade silicone coated with Rhamnolipid R89 is effective against Staphylococcus spp. biofilms. Molecules 2019, $24,3843$. [CrossRef]

9. Rosenberg, E.; Ron, E.Z. High- and low-molecular-mass microbial surfactants. Appl. Microbiol. Biotechnol. 1999, 52, 154-162. [CrossRef]

10. Chong, H.; Li, Q. Microbial production of rhamnolipids: Opportunities, challenges and strategies. Microb. Cell Fact. 2017, 16, 137. [CrossRef]

11. Ron, E.Z.; Rosenberg, E. Natural roles of biosurfactants. Minireview. Environ. Microbiol. 2001, 3, 229-236. [CrossRef] [PubMed]

12. Uzoigwe, C.; Burgess, J.G.; Ennis, C.J.; Rahman, P.K. Bioemulsifiers are not biosurfactants and require different screening approaches. Front. Microbiol. 2015, 6, 245. [CrossRef]

13. Kaskatepe, B.; Yildiz, S.; Kaskatepe, B.; Yildiz, S. Rhamnolipid Biosurfactants Produced by Pseudomonas Species. Braz. Arch. Biol. Technol. 2016, 59, 1-16. [CrossRef]

14. Laabei, M.; Jamieson, W.D.; Lewis, S.E.; Diggle, S.P.; Jenkins, A.T.A. A new assay for rhamnolipid detection-important virulence factors of Pseudomonas aeruginosa. Appl. Microbiol. Biotechnol. 2014, 98, 7199-7209. [CrossRef] [PubMed]

15. Sáenz-Marta, C.I.; de Lourdes Ballinas-Casarrubias, M.; Rivera-Chavira, B.E.; Nevárez-Moorillón, G.V. Biosurfactants as Useful Tools in Bioremediation. Adv. Bioremediat Wastewater Polluted Soil 2015. [CrossRef]

16. Pornsunthorntawee, O.; Wongpanit, P.; Chavadej, S.; Abe, M.; Rujiravanit, R. Structural and physicochemical characterization of crude biosurfactant produced by Pseudomonas aeruginosa SP4 isolated from petroleum-contaminated soil. Bioresour. Technol. 2008, 99, 1589-1595. [CrossRef]

17. Abdel-Mawgoud, A.M.; Aboulwafa, M.M.; Hassouna, N.A.-H. Characterization of Rhamnolipid Produced by Pseudomonas aeruginosa Isolate Bs20. Appl. Biochem. Biotechnol. 2009, 157, 329-345. [CrossRef]

18. Chebbi, A.; Elshikh, M.; Haque, F.; Ahmed, S.; Dobbin, S.; Marchant, R.; Sayadi, S.; Chamkha, M.; Banat, I.M. Rhamnolipids from Pseudomonas aeruginosa strain W10; as antibiofilm/antibiofouling products for metal protection. J. Basic Microbiol. 2017, 57, 364-375. [CrossRef]

19. e Silva, S.S.; Carvalho, J.W.; Aires, C.P.; Nitschke, M. Disruption of Staphylococcus aureus biofilms using rhamnolipid biosurfactants. J. Dairy Sci. 2017, 100, 7864-7873. [CrossRef]

20. Katz, H.; Farber, R.; Chaniel, G.; Ankar, Y.; Cohen, H.; Cahan, R. Rhamnolipid-enhanced Pseudomonas putida biofilm formation on hydrophilic surfaces with toluene as the bacterium's sole carbon source. Int. Biodeterior. Biodegrad. 2018, 127, 87-94. [CrossRef]

21. Sen, S.; Borah, S.N.; Bora, A.; Deka, S. Rhamnolipid exhibits anti-biofilm activity against the dermatophytic fungi Trichophyton rubrum and Trichophyton mentagrophytes. Biotechnol. Rep. 2020, 27, e00516. [CrossRef] [PubMed]

22. Viramontes-Ramos, S.; Portillo-Ruiz, M.C.; Ballinas-Casarrubias, M.d.L.; Torres-Muñoz, J.V.; Rivera-Chavira, B.E.; NevárezMoorillón, G.V. Selection of biosurfactan/bioemulsifier-producing bacteria from hydrocarbon-contaminated soil. Braz. J. Microbiol. 2010, 41, 668-675. [CrossRef] [PubMed]

23. Carrazco-Palafox, J.; Rivera-Chavira, B.E.; Ramírez-Baca, N.; Manzanares-Papayanopoulos, L.I.; Nevárez-Moorillón, G.V. Improved method for qualitative screening of lipolytic bacterial strains. MethodsX 2018, 5, 68-74. [CrossRef] [PubMed]

24. Zapién-Chavarría, K.A.; Plascencia-Terrazas, A.; Venegas-Ortega, M.G.; Varillas-Torres, M.; Rivera-Chavira, B.E.; Adame-Gallegos, J.R.; González-Rangel, M.O.; Nevárez-Moorillón, G.V. Susceptibility of Multidrug-Resistant and Biofilm-Forming Uropathogens to Mexican Oregano Essential Oil. Antibiotics 2019, 8, 186. [CrossRef]

25. Díaz De Rienzo, M.A.; Stevenson, P.S.; Marchant, R.; Banat, I.M. Pseudomonas aeruginosa biofilm disruption using microbial surfactants. J. Appl. Microbiol. 2016, 120, 868-876. [CrossRef]

26. Rikalović, M.G.; Gojgić-Cvijović, G.; Vrvić, M.M.; Karadžić, I. Production and characterization of rhamnolipids from Pseudomonas aeruginosa san-ai. J. Serbian Chem. Soc. 2012, 77, 27-42. [CrossRef]

27. Díaz De Rienzo, M.A.; Martin, P.J. Effect of Mono and Di-rhamnolipids on Biofilms Pre-formed by Bacillus subtilis BBK006. Current Microbiol. 2016, 73, 183-189. [CrossRef]

28. Rasamiravaka, T.; Vandeputte, O.; Jaziri, M. Procedure for Rhamnolipids Quantification Using Methylene-Blue. Bio-Protocol 2016, 6. [CrossRef]

29. George, S.; Jayachandran, K. Production and characterization of rhamnolipid biosurfactant from waste frying coconut oil using a novel Pseudomonas aeruginosa D. J. Appl. Microbiol. 2013, 114, 373-383. [CrossRef] 
30. Lotfabad, T.B.; Shourian, M.; Roostaazad, R.; Najafabadi, A.R.; Adelzadeh, M.R.; Noghabi, K.A. An efficient biosurfactantproducing bacterium Pseudomonas aeruginosa MR01, isolated from oil excavation areas in south of Iran. Colloid Surf. B Biointerfaces 2009, 69, 183-193. [CrossRef]

31. Santos, D.K.F.; Rufino, R.D.; Luna, J.M.; Santos, V.A.; Salgueiro, A.A.; Sarubbo, L.A. Synthesis and evaluation of biosurfactant produced by Candida lipolytica using animal fat and corn steep liquor. J. Pet. Sci. Eng. 2013, 105, 43-50. [CrossRef]

32. Nitschke, M.; Costa, S.G.V.A.O.; Haddad, R.; Gonçalves, L.A.G.; Eberlin, M.N.N.; Contiero, J.; Goncalves, L.A.G. Oil Wastes as Unconventional Substrates for Rhamnolipid Biosurfactant Production by Pseudomonas aeruginosa LBI. Biotechnol. Prog. 2005, 21, 1562-1566. [CrossRef] [PubMed]

33. Dusane, D.H.; Pawar, V.S.; Nancharaiah, Y.v.; Venugopalan, V.P.; Kumar, A.R.; Zinjarde, S.S. Anti-biofilm potential of a glycolipid surfactant produced by a tropical marine strain of Serratia marcescens. Biofouling 2011, 27, 645-654. [CrossRef]

34. Environmental Protection Agency (US-EPA). Office of Pesticide Programs. Method MB-19-05 Preparing a Pseudomonas aeruginosa or Staphylococcus aureus Biofilm using the CDC Biofilm Reactor. Last revision 01-21-20.

35. Nitschke, M.; Araújo, L.V.; Costa, S.G.; Pires, R.C.; Zeraik, A.E.; Fernandes, A.C.; Freire, D.M.; Contiero, J. Surfactin reduces the adhesion of food-borne pathogenic bacteria to solid surfaces. Lett. Appl. Microbiol. 2009, 49, 2241-2247. [CrossRef] [PubMed]

36. Wood, T.L.; Gong, T.; Zhu, L.; Miller, J.; Miller, D.S.; Yin, B.; Wood, T.K. Rhamnolipids from Pseudomonas aeruginosa disperse the biofilms of sulfate-reducing bacteria. NPJ Biofilms Microb. 2018, 4, 1-8. [CrossRef] [PubMed]

37. Elshikh, M.; Funston, S.; Chebbi, A.; Ahmed, S.; Marchant, R.; Banat, I.M. Rhamnolipids from non-pathogenic Burkholderia thailandensis E264: Physicochemical characterization, antimicrobial and antibiofilm efficacy against oral hygiene related pathogens. New Biotechnol. 2017, 36, 26-36. [CrossRef]

38. Nickzad, A.; Déziel, E. The involvement of rhamnolipids in microbial cell adhesion and biofilm development-An approach for control? Lett. Appl. Microbiol. 2014, 58, 447-453. [CrossRef]

39. Zheng, H.; Singh, N.; Shetye, G.S.; Jin, Y.; Li, D.; Luk, Y.Y. Synthetic analogs of rhamnolipids modulate structured biofilms formed by rhamnolipid-nonproducing mutant of Pseudomonas aeruginosa. Bioorg. Med. Chem. 2017, 25, 1830-1838. [CrossRef]

40. de Araujo, L.V.; Guimarães, C.R.; da Silva Marquita, R.L.; Santiago, V.M.; de Souza, M.P.; Nitschke, M.; Freire, D.M. Rhamnolipid and surfactin: Anti-adhesion/antibiofilm and antimicrobial effects. Food Control. 2016, 63, 171-178. [CrossRef]

41. do Valle Gomes, M.Z.; Nitschke, M. Evaluation of rhamnolipid and surfactin to reduce the adhesion and remove biofilms of individual and mixed cultures of food pathogenic bacteria. Food Control. 2012, 25, 441-447. [CrossRef]

42. Narayana, P.S.; Srihari, P.S. Biofilm resistant surfaces and coatings on implants: A review. Mat. Today Proc. 2019, 18, 4847-4853. [CrossRef] 